\title{
In-situ TEM Characterization of Nucleation and Growth of Nanopatterned Oxides
}

\author{
Aiming Yan*, Tao Sun* and Vinayak P. Dravid* \\ *Department of Materials Science and Engineering, Northwestern University, Evanston, IL 60208, \\ USA
}

Functional oxide ceramics have been attracting much attention for years due to their scientific and technological significance. In the recent decade, advanced nanofabrication techniques provide the capability of fabricating ceramic nanostructures with precise size and shape control, which has brought new vitality to the field of traditional ceramics [1,2]. Further development of oxide nanostructures requires fundamental understanding of the new phenomena and behavior induced by shrinking their dimension down to nanoscale under spatial constraints.

Nucleation and (grain) growth are fundamental to any phase transformation, including conversion of liquid precursor to glassy/amorphous- to eventual solid-state form; as the sol-gel [3,4]. This is particularly important in emerging nanostructures and nanoscale patterns, where spatial and dimensional constraints are expected to significantly alter the local thermodynamic and kinetic conditions. Further, a detailed understanding of the early stage of such phase transformation is critical for manipulation and tailoring of "internal" microstructure of nanostructures and nanopatterns. However, despite the universal acknowledgement and significance, relevant early stage studies of nucleation and growth are rarely reported due to the challenges in building an effective test-bed, compatible with measurements tools and techniques.

In the present study, $\mathrm{CoFe}_{2} \mathrm{O}_{4}(\mathrm{CFO})$ nanodots were fabricated using "soft" electron beam lithography (soft-eBL), a nanopatterning approach recently developed in our group [5,6]. Soft-eBL is a flexible and convenient technique for patterning site-specified, shape-controlled and threedimensionally confined solid-state nanostructures on diverse substrates. It synergistically combines the top-down with bottom-up method. By using sol-gel as precursor, oxide nanostructures prepared by soft-eBL are readily appropriate for in-situ studies of nucleation and growth phenomena.

Transmission electron microscopy (TEM) was applied to quantitatively characterize the nucleation and growth behavior of CFO nanodots, which were patterned on both electron transparent $\mathrm{SiN}_{\mathrm{X}}$ membranes and $\mathrm{MgO}$ single-crystal substrates. Electron diffraction and other analytical techniques (EDS, EELS) were utilized to identify the phase and chemical composition of early-stage nuclei. Dark field images were taken for providing statistical information of size distribution of the nuclei (small crystallites) in CFO nanodots. By employing a heating sample holder, evolution of small nuclei could be monitored in-situ and in real time, to enable unambiguous study of the thermodynamics and kinetics of nucleation and growth (See Fig. 1).

The preliminary results reveal that the activation energy for grain growth of CFO nanodots is notably different than both CFO thin films and bulk materials [7]. We speculate that the increased intrinsic hydrostatic pressure due to the larger aspect ratio of nanodots and modulated strain field induced by the substrate may be responsible for these changes. Moreover, the CFO nanodot patterned on $\mathrm{MgO}$ substrate eventually grows into epitaxial single crystal. Heterogeneous nucleation 
and preferentially grain growth are observed and regarded as the key pathway for single-crystal conversion. Given the versatility of soft-eBL approach and in-situ TEM technique, the proposed strategy should be applicable to other oxide materials. We believe such studies will facilitate elucidation of the behavior of constrained ceramic nanostructures with significant implications for their technological applications.

\section{References:}

[1] V. Nagarajan, R. Ramesh et al., , Nature Materials, 2 (2003) 43

[2] Z.W. Pan, et al., Science, 291 (2001) 1947

[3] R. W. Schwartz, Chem. Mater., 9 (1997) 2325

[4] T. Sun, V.P. Dravid et al., Phys. Rev. B, 77 (2008) 205414

[5] Z. Pan, V.P. Dravid, et al., Nano Lett., 6 (2006) 2344

[6] Z. Pan, V.P. Dravid, et al., Small, 2 (2006) 274

[7] M.J. Iqbal, Mah Rukh Siddiquah, Journal of Alloys and Compounds 453 (2008) 513-518

[8] This work was supported by the U.S. Department of Energy (DOE-BES) programs, the grant number DE-FG02-07ER46444. DOE's support does not constitute an endorsement by DOE of the views expressed in the article. The research work was performed in the EPIC facilities of the NUANCE Center at Northwestern University. NUANCE Center is supported by NSF-NSEC, NSFMRSEC, the State of Illinois, and Northwestern University.
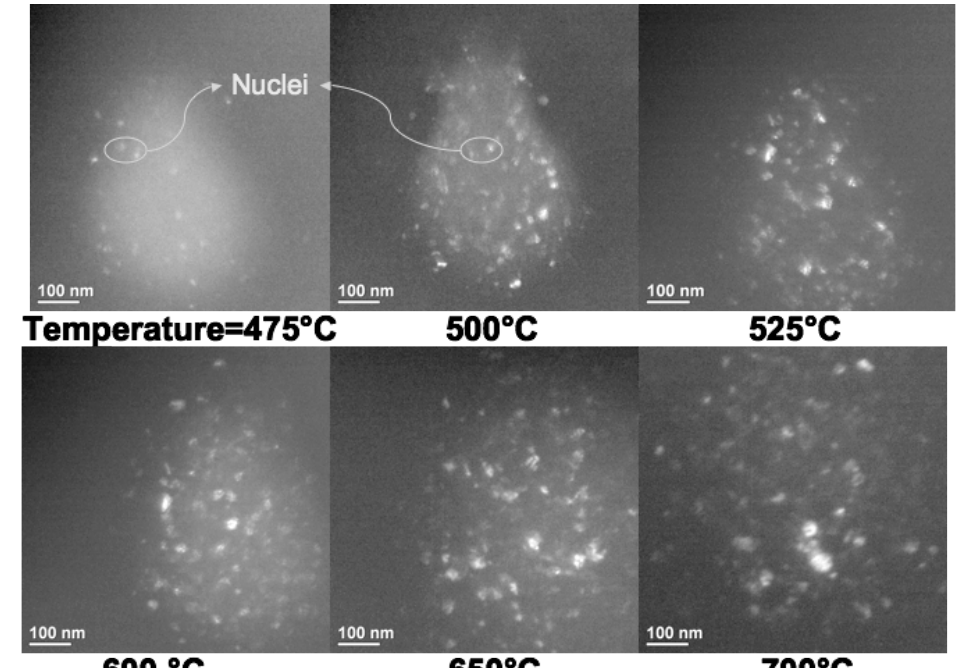

Figure 1: Dark Field Images of CFO nanodot $(250 \mathrm{~nm})$ at different temperatures. Scale bar $=100 \mathrm{~nm}$.

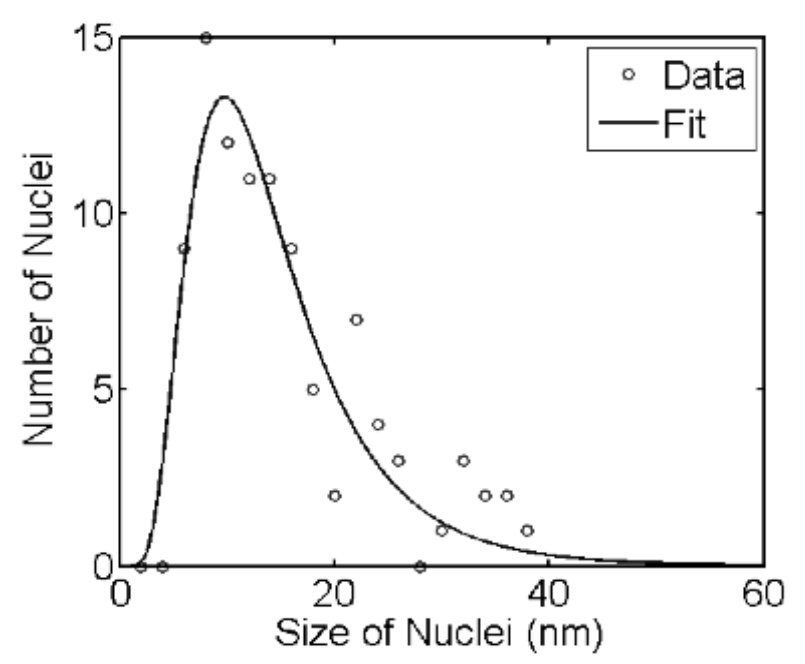

Figure 2: Lognormal fit of the nuclei size distribution at one temperature and the average nuclei size at this temperature (e.g. as shown here, nuclei in CFO nanodot at $600^{\circ} \mathrm{C}$ ).

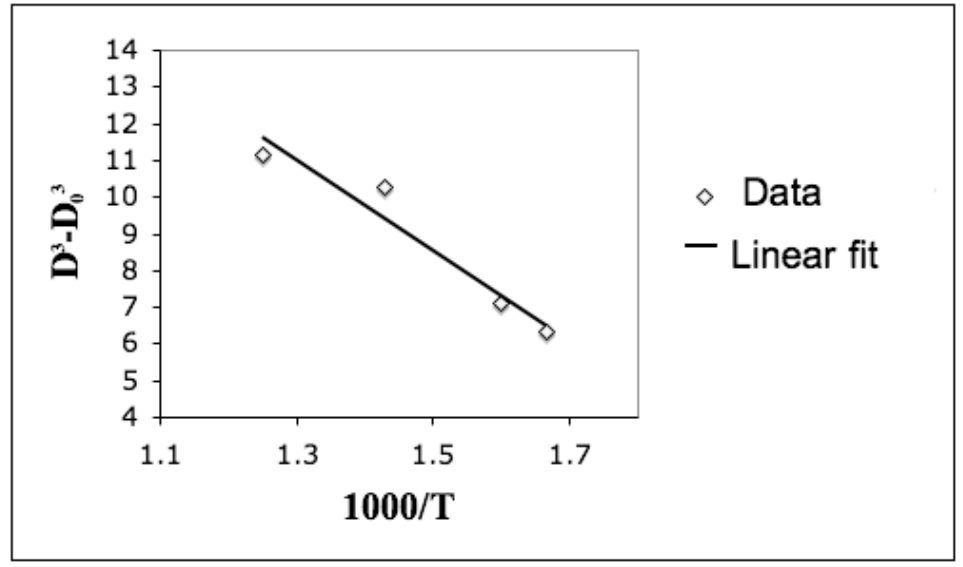

Figure 3: Activation energy calculated from the data (e.g. activation energy for a $150 \mathrm{~nm}$ CFO nanodot: Slope $\left.=2.75=-1000 * \mathrm{Ea} / \mathrm{K}_{\mathrm{B}}, \mathrm{Ea} \approx 0.24 \mathrm{eV}\right)$. 